\title{
Virtual reality versus Biodex training in adolescents with chronic ankle instability: a randomized controlled trial
}

Tamer M. Shousha ${ }^{1,2}$, Nehad A. Abo-zaid ${ }^{3}$, Hamada Ahmed Hamada ${ }^{4}$, Mohamed Y. Abdelhamid Abdelsamee ${ }^{5}$, Mohamed A. Behiry ${ }^{6}$
Corresponding author:

Hamada Ahmed Hamada

Department

of Biomechanics

Faculty of

Physical Therapy

Cairo University

Cairo, Egypt

Phone: +201117893697

E-mail: hamada.ahmed@ pt.cu.edu.eg

Submitted: 7 March 2021

Accepted: 18 March 2021

Arch Med Sci

https://doi.org/10.5114/aoms/134635

Copyright $\odot 2021$ Termedia \& Banach

\section{Abstract}

Introduction: Ankle instability is a common injury in athletes, affecting the quality of life, functional limitation, as well as static and dynamic balance. The aim of the study was to compare the efficacy of virtual reality and Biodex balance training in the treatment of ankle instability in adolescent athletes.

Material and methods: Ninety football players were enrolled in this study after the assessment of their eligibility. Their age ranged from 12 to 16 years. They were randomly allocated to three groups of equal numbers. Group 1 received a guideline protocol, group 2 received the same guideline protocol in addition to Wii Sport Training, and group 3 received the same guideline protocol in addition to Biodex balance training. All groups received treatment protocols three times a week for 3 months. Outcome measures included the stability indices (overall stability, anteroposterior stability, and mediolateral) measured by the Biodex Balance System as well as ankle instability measured by the Cumberland Ankle Instability Tool. Measurements were collected at the baseline and after 3 months of treatment.

Results: Post-treatment findings revealed a statistically significant decrease in the overall, anteroposterior, and mediolateral stability indices, as well as significant increase in Cumberland Ankle Instability outcomes of all groups $(p<0.05)$. On the other hand, there were no statistically significant differences between the virtual reality and Biodex balance training groups.

Conclusions: Virtual reality training has a significant effect which appears to be similar to the Biodex balance training in adolescent athletes with chronic ankle instability.

Key words: ankle, instability, adolescent, virtual reality, Biodex, balance. 


\section{Introduction}

Ankle and foot injuries are the most common pediatric athlete injuries [1] with a prevalence of 2.15 per 1000 [2]. It was also reported that the prevalence was $0.7 \%$ and $0.4 \%$ for mild and severe chronic ankle instabilities (CAl) among males and $0.3 \%$ and $0.4 \%$ among females, respectively, whereas males had a 2.33-fold greater incidence of mild CAI than females [3]. Ankle sprains (AS) are the most frequent sports-related injuries among athletes and physically active individuals [2]. Individuals with a history of AS are more susceptible to chronic ankle instability [4]. The CAI can be presented as functional instability perceived as giving way, or as mechanical instability that results from ligamentous and pericapsular laxity [5]. Ankle instability (Al), limited mobility of the ankle joint, muscle weakness, and proprioceptor damage in the ankle ligaments are the most common risk factors reported. The injury results from overstretching the ligaments that support the ankle joint beyond their physiological limits, leading to damage of the ligaments fibrous integrity [6]. Incomplete healing of injuries in lateral ligaments of the ankle can lead to an increase in the accessory movements causing an enlargement of the neutral zone and an abnormal pattern of joint movement [7]. The injury does not solely affect the ligamentous integrity, but extends to include related joint structures. Recent ultrasonic evaluations reported the cross sectional area of the peroneus longus to be reduced in patients with lateral AS [8].

Functional ankle instability results in altered activation patterns of the lower limb proximal muscles reflected as significant differences in amplitude of proximal muscle activity with distal muscle activity during perturbation [9].

According to McKeon, lateral AS and CAI lead to muscle injury and peripheral neural structures, central neural inhibition and sensory reorganization, and neuromotor impairment providing impaired postural control and balance [10].

In addition, deficits in postural control resulting from impaired neuro-motor control and proprioception are commonly found in patients with CAI [11], whereas various balance-training programs were reported to improve postural control in patients with CAI [12].

Several treatment protocols were reported to improve ankle stability including proprioceptive [13], coordination [14], balance [15], strength, and virtual reality training [16].

Virtual reality (VR) gaming is a new technology, enhancing and providing multiple innovative in terventions that require attention and active participation from patients [17]. It gives a chance to have interaction in multisensory activities that are almost like existence including hand skills, activi- ties of daily living, manipulation, mobility, balance, and cognition [18]. The Nintendo Wii Fit Plus is a common designed VR used to improve balance and fitness while providing entertainment. It has a great impact on the improvement of functional performance by enhancing flexibility, balance, strength, and coordination training [19].

On the other hand, the Biodex balance training (BBT) system can improve the functional abilities of the neuro-musculoskeletal system by decreasing the malalignment of posture and improving spinal kinematics and balance control [20]. To date, several studies have been reported comparing VR and BBT in cases of AS, but to our knowledge, none has addressed adolescent athletes. Therefore, the purpose of this study was to compare the effect of virtual reality training (VRT) in the form of Wii Fit Plus games and BBT for ankle instability in adolescent athletes. It was hypothesized that there is a significant difference between the efficacy of VRT and BBT in rehabilitation of ankle instability in adolescent athletes.

\section{Material and methods}

\section{Study design}

A randomized controlled study was conducted in an outpatient private clinic, during the period from August 2018 to August 2020. An informed consent form was signed by the parent of each participant. Verbal assent was obtained from each participant in the presence of the parent or legal guardian. Procedures of our study had approval No. P.T.REC/012/002877 from the Institutional Ethical Committee Clearance of the Faculty of Physical Therapy, Cairo University, and had registration number NCT04592900 that was registered at Clinicaltrial.gov.

\section{Confidentiality}

All research procedures from the orientation to the actual application of the study were conducted in a closed room in the private outpatient clinic. We ensured that no one other than the investigators was present in the room during the study.

\section{Declaration of interests}

No interest other than the information gained and data obtained from participants for the research study.

\section{Access to data}

The participants' personal data and information were saved in an Excel spreadsheet that was only accessible to the research investigators. The sheet was on the primary investigator's computer that is secured by a password. 


\section{Ancillary and post-trial care}

The study does not involve any physical, psychological or social risks. The study could be stopped if any unexpected symptoms were reported by the patients during treatment.

\section{Dissemination policy}

Reports and results were sent by email to each participant parent/guardian individually.

\section{Participants}

Ninety male football players who were diagnosed with Al based on a careful clinical evaluation were referred by qualified physicians of at least 10 years of experience and confirmed by an experienced physiotherapist of at least 10 years of experience as well.

The study inclusion was restricted to males who sustained a lateral ankle sprain of grade II, in the age range of $12-16$ years $(15.23 \pm 1.21)$, with a history of [21]:

1. At least one significant ankle sprain where:

- The initial sprain occurred at least 12 months prior to study enrollment.

- It caused at least 1 interrupted day of desired physical/sport activity.

- The most recent injury occurred more than 3 months prior to study enrollment.

2. A history of the previously injured ankle joint "giving way", and/or recurrent sprain, and/or "feelings of instability".

Participant also had to obtain a score of 24 or less according to the Cumberland Ankle Instability Tool (CAIT), and had a stable medical and psychological status as reported by the team physician. The authors excluded from the current study participants with grades I and III ankle sprain, ankle or foot deformity, back problems, or any musculoskeletal disorders; a history of previous surgery on the musculoskeletal structures (i.e., bones, joint structures, nerves) in either lower extremity, acute injury to musculoskeletal structures of other joints of the lower extremity in the previous 3 months that impacted joint integrity and function resulting in at least 1 interrupted day of desired physical activity, any disorder affecting balance, still receiving ankle physical therapy treatment, or did not have any previous exposure to exergaming (the activity of playing video games that involve physical exertion and are thought of as a form of exercise) [22]. Participants who did not commit to two successive sessions were also considered for exclusion.

\section{Randomization}

One hundred athletic adolescents with CAI were assessed for eligibility. Six did not meet our selected criteria, one did not agree to enroll, and three dropped out from post-assessment. Ninety children were assigned randomly to three groups of equal numbers. Randomization was limited to different sizes of permuted blocks to ensure the allocation of equal numbers in each group. Each random permuted block was stored in a drawer locked until required in the form of sealed consecutive sequence, numbered, opaque envelopes. After the formal inclusion of each participant in the trial and in the presence of the patient's parent, the next envelope was opened by the researcher in sequence. All participants were blinded to the study hypothesis. A randomization diagram of participants is shown in Figure 1.

\section{Outcome measures}

\section{Primary: CAIT}

It is a self-reporting scale that assesses the degree of Al. It consists of 30 points of a total of nine items. Recording at least a 28-point score is defined as a subject having a normal ankle, while those with a point score of 24 or less are defined as subjects having functional ankle instability (FAI). A higher score indicates greater ankle stability [23].

CAIT is reported as a simple, valid, and reliable tool to assess the severity of functional ankle instability. The CAIT score threshold was reported as 27.5 (Youden index, 68.1 ) with sensitivity of $82.9 \%$ and specificity of $74.7 \%$. Test-retest reliability was excellent; $\operatorname{ICC}(2,1)=0.96$ [24].

All measurements were collected at the baseline and after 3 months of treatment.

\section{Secondary: Postural stability}

The Biodex Balance System (BBS) SD (Model 950-441) is an important therapeutic tool used for the assessment and training of patients with deficits in balance [25]. The BBS is a reliable and valid device used to evaluate the participant's ability to maintain static and dynamic balance stability on the unstable tilting platform; clinicians can evaluate postural control by measuring the ability to maintain dynamic unilateral and bilateral posture stability on the unstable surface. During the posture stability test, we evaluate the variance from the center by measuring the ability of the participant to control the platform's angle of tilt [26]. A dynamic balance test was performed on stability level eight according to a prior pilot study. The outcome measures were: the overall stability index (OASI), which refers to the ability of the participant to maintain his balance in all directions; the antero-posterior stability index (APSI), which refers to the participant's ability to maintain his balance from forward to backward direction; and 


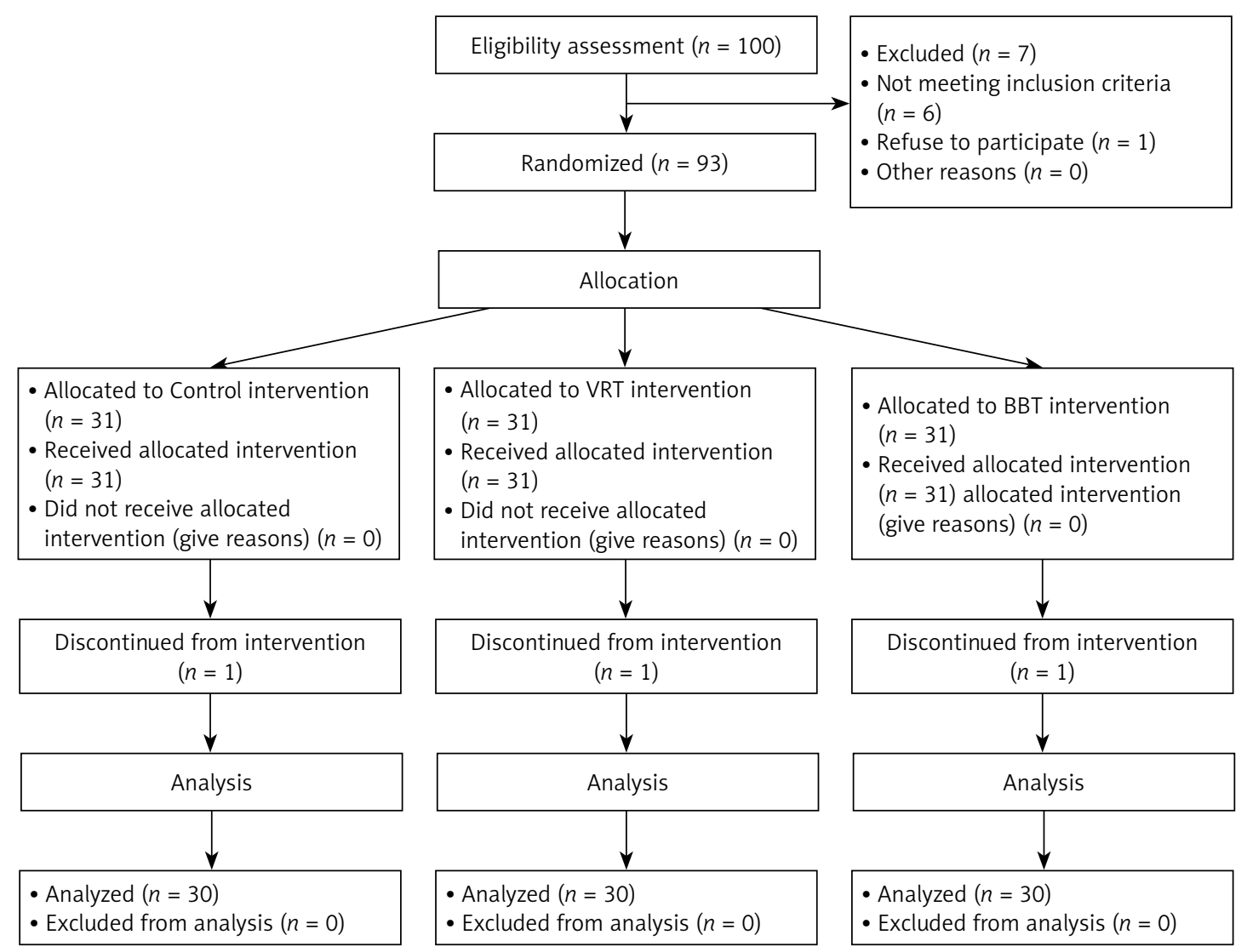

Figure 1. Randomization flow chart

the medio-lateral stability index (MLSI), which refers to the ability of the participant to maintain his balance from side-to-side directions. High values indicate that the participant had difficulty.

The Biodex stability system is actually a movable platform with variable degrees of instability (static, from 1 to 12). The platform can be tilted up to twenty degrees of surface tilt from horizontal in all directions. It has a foot grid for determination of foot position before testing. It has 12 stability levels. Stability level 12 is the most stable platform surface, whereas stability level 1 is the most unstable platform surface [27].

\section{Intervention}

Intervention was provided individually in a face-to-face mode of delivery by a therapist with at least 15 years of clinical experience in ankle and foot rehabilitation.

The Biodex was chosen based on being the most widely used in our region as well as based on its validity, reliability and ease of use. Meanwhile, the Wii Fit was chosen since active video gaming has become an emerging trend in fitness, education and health sectors [22]. It was also reported as a safe and feasible adjunct to physiotherapy [28], increasing activity levels, vitality and well-being while being fun [29].
In addition, it can be used independently at home, especially during a pandemic [30].

- The control group received 60 min of a guideline protocol that was adopted from previous proprioceptive and balance rehabilitation programs [31, 32].

Proprioceptive training was based on the participant's ability to maintain a single-limb stance while performing various tasks. The exercise sequence was (1) hop to stabilization, (2) hop to stabilization and reach, (3) hop-to-stabilization box drill, (4) progressive single-limb stance activities with eyes open, and (5) progressive single-limb stance activities with eyes closed. Each participant progressed to the next level only after completing the previous level without faults. Faults were considered when touching down with the contralateral limb, trunk bending more than $30^{\circ}$ to either side, removing the hands from the hip level during hands-on-hips exercises, bracing the non-weightbearing extremity against the weight-bearing one, or missing the target.

Thera Band resistance was also used to strengthen plantar flexors, dorsiflexors, invertors, and evertor muscles. Additional balance exercises were performed by perturbation on a slide board, Airex Balance Pad, trampoline, and donut hall [32]. - The VR group received $30 \mathrm{~min}$ of the same guide- 
line protocol in addition to 30 min VR. Nintendo Wii Fit Plus is one of the most common homebased interactive video games (IVG), consisting of a wireless handheld pointing device, the detection of movement and acceleration being in three dimensions by a motion sensor attached to a television. The virtual image is seen on the television screen that mimics the participant's movements. These games encourage the interaction of participants to play repetitively [33]. The strength and balance exercises were carefully selected by physical therapists to provide an optimum effect.

- The guideline of the VRT protocol was timed and calculated by the program and displayed on the screen. The program included: lunge, single leg extension, sideways leg lift, single leg twist, and rowing squat as strength exercises; and soccer heading, tight rope walks, ski slalom, table tilt, and snowboard slalom as balance exercises [16].

- The BBT group received 30 min of the same guideline protocol of assessment of balance on the BBS [34].

- All participants received treatment three times/ week for 3 consecutive months.

\section{Sample size}

To determine the required number of subjects needed in this study, estimates of mean and standard deviation were collected from a pilot study consisting of 10 patients who received the same program between June 1, 2018 and July 30, 2018. The mean differences and standard deviation of the CAIT score were estimated as 0.52 and 3.4, respectively, from this pilot study. These findings are consistent with Wright et al. [35], who identified the minimal detectable change (MDC) and minimal clinically important difference (MCID) as 3.08 and $>3$ respectively. According to these figures, at least 25 participants per group, given a significance level of $5 \%$ and statistical power of $80 \%$, were needed in the current study. To account for possible participant drop-outs, the sample size was increased by $20 \%$. Calculations were performed using the ps power and sample size calculation computer program available online from the Vanderbilt Medical Center (www. mc.vanderbilt.edu). The sample size was based on the expected difference in the primary outcome variable between the groups at post-test only.

\section{Statistical analysis}

The data were screened before the final analysis to test the normality assumption by using the Shapiro-Wilk test, which reflects normal distribution $(p>0.05)$. Additionally, Levene's test was used to testing the homogeneity of variance, which revealed no significant difference $(p>0.05)$. Since data were normally distributed, parametric analysis was used. The SPSS package program version 25 for Windows (SPSS, Inc., Armonk, NY: IBM Corp) was used. All data were expressed as mean and standard deviation for demographic data, balance measurement, and ankle instability measurement. A mixed design $3 \times 2$ MANOVA-test was used to compare the tested variables in different groups and measuring periods. The first independent variable (between-subject factors) was the tested group with 3 levels (control group, VR group, and BBT group). The second independent variable (within-subject factor) was the testing time before and after treatment. The four dependent variables were the OASI, APSI, MLSI, and CAIT. The Bonferroni correction test was used to compare between pairs of groups for post-treatment of the tested variables whose F-value was significant in the MANOVA test. The significance level was set at $p \leq 0.05$.

\section{Results}

Ninety patients participated in this study and were distributed randomly as 30 patients in each group. No significant differences in age, weight, height, and BMI were found among the three groups (Table I).

Multivariate tests by $3 \times 2$ mixed design MANOVA for outcome measures presented statistically significant $(p<0.05)$ effects due to main effects of tested groups (F17.487; $p=0.0001$; partial $\left.\eta^{2}=0.290\right)$, measurement time $(F=225.796 ; p=$

Table I. Comparison of mean values of demographic data among three groups

\begin{tabular}{|c|c|c|c|c|}
\hline \multirow[t]{2}{*}{ Variables } & \multicolumn{3}{|c|}{ Groups (mean $\pm S D$ ) } & \multirow[t]{2}{*}{$P$-value } \\
\hline & $\begin{array}{l}\text { Control group } \\
\quad(n=30)\end{array}$ & $\begin{array}{l}\text { VR group } \\
(n=30)\end{array}$ & $\begin{array}{l}\text { BBT group } \\
(n=30)\end{array}$ & \\
\hline Age [years] & $15.23 \pm 0.84$ & $15.74 \pm 0.95$ & $15.32 \pm 0.92$ & 0.073 \\
\hline Weight [kg] & $56.27 \pm 2.74$ & $56.34 \pm 2.76$ & $55.93 \pm 2.67$ & 0.828 \\
\hline Height $[\mathrm{cm}]$ & $160.51 \pm 6.86$ & $159.34 \pm 7.06$ & $160.93 \pm 7.13$ & 0.662 \\
\hline $\mathrm{BMI}\left[\mathrm{kg} / \mathrm{m}^{2}\right]$ & $21.84 \pm 1.90$ & $22.19 \pm 2.12$ & $21.60 \pm 2.19$ & 0.546 \\
\hline
\end{tabular}


0.0001 ; partial $\left.\eta^{2}=0.841\right)$, and group $x$ time interaction $\left(F=342.00 ; p=0.0001\right.$; partial $\left.\eta^{2}=0.245\right)$.

The statistical analysis within each group (Table II) revealed a significant $(p<0.05)$ decrease in OASI, APSI, and MLSI at post-treatment compared to pre-treatment within the control, VR, and BBT groups. Measurement of the ankle instability (CAIT) significantly $(p<0.05)$ increased at post-treatment compared to pre-treatment within the control group, VR group, and BBT group. Both the VR group and the BBT group revealed more improvement in postural balance and CAIT than the control group.

The statistical analysis between the 3 groups (Table II) showed no significant differences $(p>0.05)$ in mean values of pre-treatment OASI,
APSI, MLSI, and CAIT. However, there were significant differences $(p<0.05)$ in the mean values of post-treatment OASI, APSI, MLSI, and CAIT between the three groups.

From post-hoc test comparisons (Table III), there was a significant difference $(p<0.05)$ between the control and VR groups, control, and BBT groups, whereas no significant differences were found $(p>0.05)$ between VR and BBT groups for measurements of balance and CAIT.

\section{Discussion}

The current trial aimed to compare the efficacy of VR and BBT in the treatment of CAI in ado-

Table II. Comparison by MANOVA test for all measured dependent variables within and among three groups

\begin{tabular}{|c|c|c|c|c|}
\hline \multirow[t]{2}{*}{ Variables } & \multicolumn{3}{|c|}{ Groups (mean $\pm S D$ ) } & \multirow[t]{2}{*}{$P$-value } \\
\hline & $\begin{array}{l}\text { Control group } \\
\quad(n=30)\end{array}$ & $\begin{array}{l}\text { VR group } \\
(n=30)\end{array}$ & $\begin{array}{l}\text { BBT group } \\
(n=30)\end{array}$ & \\
\hline \multicolumn{5}{|l|}{ OASI: } \\
\hline Pre-treatment & $2.41 \pm 0.31$ & $2.35 \pm 0.28$ & $2.30 \pm 0.37$ & 0.405 \\
\hline Post-treatment & $2.01 \pm 0.42$ & $1.22 \pm 0.19$ & $1.16 \pm 0.26$ & $0.0001^{*}$ \\
\hline Mean difference & 0.40 & 1.13 & 1.14 & \\
\hline Improvement \% & 16.60 & 48.09 & 49.57 & \\
\hline$P$-value & $0.0001^{*}$ & $0.0001^{\star}$ & $0.0001^{*}$ & \\
\hline \multicolumn{5}{|l|}{ APSI: } \\
\hline Pre-treatment & $1.61 \pm 0.19$ & $1.55 \pm 0.16$ & $1.60 \pm 0.28$ & 0.469 \\
\hline Post-treatment & $1.24 \pm 0.27$ & $0.77 \pm 0.13$ & $0.78 \pm 0.18$ & $0.0001^{*}$ \\
\hline Mean difference & 0.37 & 0.78 & 0.82 & \\
\hline Improvement \% & 22.98 & 50.32 & 51.25 & \\
\hline$P$-value & $0.0001^{*}$ & $0.0001^{*}$ & $0.0001^{*}$ & \\
\hline \multicolumn{5}{|l|}{ MLSI: } \\
\hline Pre-treatment & $1.45 \pm 0.36$ & $1.41 \pm 0.31$ & $1.39 \pm 0.39$ & 0.850 \\
\hline Post-treatment & $1.29 \pm 0.63$ & $1.08 \pm 0.50$ & $1.10 \pm 0.50$ & $0.027^{\star}$ \\
\hline Mean difference & 0.16 & 0.33 & 0.29 & \\
\hline Improvement \% & 11.03 & 23.40 & 20.90 & \\
\hline$P$-value & 0.024 & $0.004^{\star}$ & $0.014^{*}$ & \\
\hline \multicolumn{5}{|l|}{ CAIT: } \\
\hline Pre-treatment & $18.50 \pm 1.49$ & $18.28 \pm 1.10$ & $18.31 \pm 2.50$ & 0.871 \\
\hline Post-treatment & $19.89 \pm 1.54$ & $22.55 \pm 1.36$ & $22.93 \pm 3.13$ & $0.0001^{*}$ \\
\hline Mean difference & 1.39 & 3.97 & 4.62 & \\
\hline Improvement \% & $7.51 \%$ & $21.72 \%$ & $25.23 \%$ & \\
\hline$P$-value & $0.001^{*}$ & $0.0001^{*}$ & $0.0001^{*}$ & \\
\hline
\end{tabular}


Table III. Post-hoc pairwise test between control, VR, and BBT groups

\begin{tabular}{|c|c|c|c|}
\hline \multirow{2}{*}{ Variables } & \multicolumn{3}{|c|}{ Post-hoc (Bonferroni test) } \\
\hline & Control vs. VR & Control vs. BBT & VR vs. BBT \\
\hline \multicolumn{4}{|l|}{ Post OASI: } \\
\hline Mean difference & 0.78 & 0.81 & 0.04 \\
\hline $95 \% \mathrm{Cl}$ & $0.58-0.97$ & $0.62-1.01$ & $-0.15-0.23$ \\
\hline$P$-value & $0.0001^{*}$ & $0.0001^{*}$ & 1.000 \\
\hline \multicolumn{4}{|l|}{ Post APSI: } \\
\hline Mean difference & 0.47 & 0.44 & 0.03 \\
\hline $95 \% \mathrm{Cl}$ & $0.34-0.56$ & $0.31-0.56$ & $-0.09-0.15$ \\
\hline$P$-value & $0.0001^{*}$ & $0.0001^{*}$ & 1.000 \\
\hline \multicolumn{4}{|l|}{ Post MLSI: } \\
\hline Mean difference & 0.21 & 0.19 & 0.02 \\
\hline $95 \% \mathrm{Cl}$ & $-0.13-0.55$ & $-0.15-0.53$ & $-0.36-0.33$ \\
\hline$P$-value & 0.004 & 0.004 & 1.000 \\
\hline \multicolumn{4}{|l|}{ Post CAIT: } \\
\hline Mean difference & 3.65 & 4.03 & 0.38 \\
\hline $95 \% \mathrm{Cl}$ & $-2.8-5.02$ & $-2.66-5.40$ & $-1.74-0.98$ \\
\hline$P$-value & $0.0001^{*}$ & $0.0001^{*}$ & 1.000 \\
\hline
\end{tabular}

$\mathrm{Cl}$ - confidence interval, *significant $(p<0.05)$, CAIT - Cumberland Ankle Instability Tool, OASI - overall stability index, APSI - anteroposterior stability index, MLSI - medio-lateral stability index, VR - virtual reality, BBT - Biodex balance training.

lescent athletes. The results of the trial showed a significant decrease in OASI, APSI, and MLSI as well as a significant increase in CAIT within both groups. However, there was no significant difference between the VR and BBT groups with regards to the outcomes of the rehabilitation for athletic adolescents with CAI.

As reported earlier, ankle sprains are common in young athletes, representing up to $30 \%$ of all sports injures [36]. This is supported by the previous finding that adolescents were at greater risk of AS than adults [37]. Consequently, CAI could affect the performance of young athletes in daily life activities.

The current findings revealed significant improvement in stability indices. This is in agreement with previous research reporting balance deficits due to impairments in proprioception and neuromuscular control $[5,38,39]$. Decreased balance in the pre-treatment assessment of all participants was directly related to the diagnosis of AS; this supports the fact that AS leads to deficits in proprioception and affects balance by increasing body sway [40]. In addition, a deficit in ROM of ankle dorsiflexion secondary to (AS) could potentially affect dynamic balance in individuals with CAI [10].

Since the balancing process requires coordination of multiple motor, sensory, and biomechani- cal components [41], it is considered an essential component of precise and functional interaction with the surrounding environment.

This explains the within-group improvements following both balance training [32, 42, 43] and conventional physiotherapy [12, 44, 45].

Additionally, this study utilized the CAIT as a reliable tool that has been widely used to assess FAI [46]. The results of the current study showed that there was a significant increase of CAIT score in the post-assessment mean values as compared to pre-assessment mean values in all groups. These results are similar to the findings of O'Driscoll et al., indicating significant improvement in CAIT score after 6 weeks of VR training in functional ankle instability; thus, increasing the CAIT score via intervention may have considerable significance to reduce $\mathrm{FAI}$ [47].

The results of the current study also showed a significant decrease in post-treatment mean values of OASI, APSI, and MLSI in the control group that received a traditional program. This is attributed to the reported positive impact of conventional physiotherapy (proprioceptive training) on the improvement of balance in patients with functional Al reducing recurrent ankle sprains [48].

Regarding virtual training, VR games such as Nintendo Wii Fit Plus have a low cost and could 
be available in the department of physiotherapy in many developing countries. It has great benefits in the rehabilitation process in areas with limited resources. The Interactive Rehabilitation Exercise System is an expensive and inaccessible VR system for the majority of the population. Therefore, it is becoming popular to use mainstream game consoles for treatment [49].

The current findings revealed a significant improvement in the VR group mainly in APSI. This reflects an increased dorsiflexion and plantar flexion (DF/PF) strength ratio [50].

According to Coughlan, \& Caulfield, VR in the form of Wii Fit Plus training is considered neuromuscular training involving balanced exercises and closed chain exercises which has a positive impact on balance in athletic patients [11]. It was concluded that Wii exercises had a great impact on improving muscle strength and balance (i.e., static, and dynamic balance) in patients with functional Al [11, 16, 32].

Similarly, improvement in means values of the stability indices OASI, APSI, and MLSI in the BBT group may be attributed considering BBT as a form of closed kinetic chain exercises that can stimulate muscle and joint mechanoreceptors and facilitate agonists and antagonists' co-contraction that help to regain balance, postural control and produce better functional joint stability $[42,51]$.

Comparison of mean values of the stability index between conventional therapy and VR training showed significant improvement of all stability indices - OASI, APSI, and MLSI - in favor of VR, similar to previous findings revealing significant improvement of static and dynamic balance in subjects with functional ankle instability [16].

Comparison of mean values of the stability index between conventional therapy and BBT training showed significant improvement of all stability indices - OASI, APSI, and MLSI - in favor of BBT. Similar findings were also reported by Rozzi et al., who concluded that BBT is an effective way to improve proprioception of joint and ability of subjects with Al to stand on a single leg [27].

The present study showed that there was no significant difference in the post-treatment mean values of the stability index between the VR group and BBT group. Our findings are in agreement with Khalil et al., who reported that there was no significant difference between the VR group and BBT group after 4 weeks of training in normal adults [50].

The current findings did not agree with Punt et al., who found no significant difference between the Wii Fit Plus exercise as a form of VR and conventional physical therapy programs in patients with an ankle sprain [52]. Also, Vernadakis et al. concluded that there was no significant difference in all stability index variables between VR and conventional therapy [53]. We believe the disagreement in the findings may be due to the difference in the population age as well as the duration of applying the intervention.

This study was conducted only on male participants; thus it did not consider the effect of gender.

Further studies are required to evaluate the efficacy of application for longer durations and allowing a follow-up period to ensure our statistical findings over time. We also recommend repeating the study on adolescent female athletes to reveal any possible gender differences in the relevant outcomes.

In conclusion, the findings of our study showed that both VR and BBT have an evident therapeutic effect on improving the degree of balance in cases of CAI in adolescent athletes but with no significant difference observed between VR and BBT during the rehabilitation of athletic adolescents with CAl.

\section{Acknowledgments}

The authors would like to express their gratitude to all participants committed to this study during this critical period.

\section{Conflict of interest}

The authors declare no conflict of interest.

\section{References}

1. Krogh-Madsen R, Thyfault JP, Broholm C, et al. A 2-wk reduction of ambulatory activity attenuates peripheral insulin sensitivity. J Appl Physiol 2010; 108: 1034-40.

2. Waterman BR, Owens BD, Davey S, Zacchilli MA, Belmont Jr PJ. The epidemiology of ankle sprains in the United States. JBJS 2010; 92: 2279-84.

3. Hershkovich O, Tenenbaum S, Gordon B, et al. A largescale study on epidemiology and risk factors for chronic ankle instability in young adults. J Foot Ankle Surg 2015; 54: 183-7.

4. Wright IC, Neptune RR, van den Bogert AJ, Nigg BM. The influence of foot positioning on ankle sprains. J Biomech 2000; 33: 513-9.

5. Hertel J. Functional anatomy, pathomechanics, and pathophysiology of lateral ankle instability. J Athl Train 2002; 37: 364.

6. van Ochten JM, De Vries AD, Van Putte N, et al. Association between patient history and physical examination and osteoarthritis after ankle sprain. Int I Sports Med 2017; 38: 717-24.

7. Panjabi MM. The stabilizing system of the spine. Part II. Neutral zone and instability hypothesis. J Spinal Disord 1992; 5: 390.

8. Lobo CC, Morales CR, Sanz DR, Corbalán IS, Marín AG, López DL. Ultrasonography comparison of peroneus muscle cross-sectional area in subjects with or without lateral ankle sprains. J Manipulative Physiol Ther 2016; 39: 635-44.

9. Kazemi K, Arab AM, Abdollahi I, López-López D, Calvo-Lobo C. Electromiography comparison of distal and proxi- 
mal lower limb muscle activity patterns during external perturbation in subjects with and without functional ankle instability. Hum Mov Sci 2017; 55: 211-20.

10. Hoch MC, Staton GS, McKeon JMM, Mattacola CG, MCKeon PO. Dorsiflexion and dynamic postural control deficits are present in those with chronic ankle instability. J Sci Med Sport 2012; 15: 574-9.

11. Coughlan G, Caulfield B. A 4-week neuromuscular training program and gait patterns at the ankle joint. J Athl Train 2007; 42: 51-9.

12. Hale SA, Hertel J, Olmsted-Kramer LC. The effect of a 4-week comprehensive rehabilitation program on postural control and lower extremity function in individuals with chronic ankle instability. J Orthop Sport Phys Ther 2007; 37: 303-11.

13. Lee D, Han S. Relationship between hip joint position sense and balance abilities. J Korean Soc Precis Eng 2018; 35: 1205-9.

14. Ross SE, Guskiewicz KM. Effect of coordination training with and without stochastic resonance stimulation on dynamic postural stability of subjects with functional ankle instability and subjects with stable ankles. Clin J Sport Med 2006; 16: 323-8.

15. Youssef NM, Abdelmohsen AM, Ashour AA, Elhafez NM, Elhafez SM. Effect of different balance training programs on postural control in chronic ankle instability: a randomized controlled trial. Acta Bioeng Biomech 2018; 20.

16. Kim KJ, Jun HJ, Heo M. Effects of Nintendo Wii Fit Plus training on ankle strength with functional ankle instability. J Phys Ther Sci 2015; 27: 3381-5.

17. Tanner L. Doctors use Wii games for rehab therapy: USA Today. Associated Press McLean, VA 2008.

18. Brütsch K, Schuler T, Koenig A, et al. Influence of virtual reality soccer game on walking performance in robotic assisted gait training for children. J Neuroeng Rehabil 2010; 7: 15

19. Williams MA, Soiza RL, Jenkinson AM, Stewart A. EXercising with $C$ omputers in $L$ ater $L$ ife (EXCELL)-pilot and feasibility study of the acceptability of the Nintendo ${ }^{\oplus}$ Wiifit in community-dwelling fallers. BMC Res Notes 2010; 3: 238.

20. El-Gohary TM, Emara HA, Al-Shenqiti A, Hegazy FA. Biodex balance training versus conventional balance training for children with spastic diplegia. J Taibah Univ Med Sci 2017; 12: 534-40.

21. Delahunt E, Coughlan GF, Caulfield B, Nightingale EJ, Lin CWC, Hiller CE. Inclusion criteria when investigating insufficiencies in chronic ankle instability. Med Sci Sports Exerc 2010; 42: 2106-21.

22. Benzing V, Schmidt M. Exergaming for children and adolescents: strengths, weaknesses, opportunities and threats. J Clin Med 2018; 7: 422.

23. Kim KJ, Choi BJ, Choi HJ, Yu SH, Kim YE. The comparison of balance using Cumberland ankle instability tool to stable and instability ankle. J Korean Soc Phys Med 2013; 8: 361-8.

24. Hiller CE, Refshauge KM, Bundy AC, Herbert RD, Kilbreath SL. The cumberland ankle instability tool: a report of validity and reliability testing. Arch Phys Med Rehabil 2006; 87: 1235-41.

25. El-Shamy SM, Abd El Kafy EM. Effect of balance training on postural balance control and risk of fall in children with diplegic cerebral palsy. Disabil Rehabil 2014; 36: 1176-83.

26. Arifin N, Osman NAA, Abas WABW. Intrarater test-retest reliability of static and dynamic stability indexes mea- surement using the Biodex Stability System during unilateral stance. J Appl Biomech 2014; 30: 300-4.

27. Rozzi SL, Lephart SM, Sterner R, Kuligowski L. Balance training for persons with functionally unstable ankles. J Orthop Sport Phys Ther 1999; 29: 478-86.

28. McPhail SM, O'Hara M, Gane E, Tonks P, Bullock-Saxton J, Kuys SS. Nintendo Wii Fit as an adjunct to physiotherapy following lower limb fractures: preliminary feasibility, safety and sample size considerations. Physiotherapy 2016; 102: 217-20.

29. Thomas S, Fazakarley L, Thomas PW, et al. Mii-vitaliSe: a pilot randomised controlled trial of a home gaming system (Nintendo Wii) to increase activity levels, vitality and well-being in people with multiple sclerosis. BMJ Open 2017; 7: e016966.

30. Gao Z, Lee JE, McDonough DJ, Albers C. Virtual reality exercise as a coping strategy for health and wellness promotion in older adults during the COVID-19 pandemic. J Clin Med 2020; 9: 1986.

31. Mckeon PO, Ingersoll CD, Kerrigan DC, Saliba E, Bennett BC, Hertel J. Balance training improves function and postural control in those with chronic ankle instability. Med Sci Sports Exerc 2008; 40: 1810-9.

32. Kim KJ, Heo M. Comparison of virtual reality exercise versus conventional exercise on balance in patients with functional ankle instability: a randomized controlled trial. J Back Musculoskelet Rehabil 2019; 32: 905-11.

33. Lohse KR, Hilderman CGE, Cheung KL, Tatla S, Van der Loos HFM. Virtual reality therapy for adults post-stroke: a systematic review and meta-analysis exploring virtual environments and commercial games in therapy. PLoS One 2014; 9: e93318.

34. Arnold BL, Schmitz RJ. Examination of balance measures produced by the biodex stability system. J Athl Train 1998; 33: 323-7.

35. Wright CJ, Linens SW, Cain MS. Establishing the minimal clinical important difference and minimal detectable change for the cumberland ankle instability tool. Arch Phys Med Rehabil 2017; 98: 1806-11.

36. Pommering TL, Kluchurosky L, Hall SL. Ankle and foot injuries in pediatric and adult athletes. Prim Care 2005; 32: 133-61.

37. Doherty C, Delahunt E, Caulfield B, Hertel J, Ryan J, Bleakley C. The incidence and prevalence of ankle sprain injury: a systematic review and meta-analysis of prospective epidemiological studies. Sport Med 2014; 44: 123-40.

38. Terada M, Bowker S, Thomas AC, et al. Alterations in stride-to-stride variability during walking in individuals with chronic ankle instability. Hum Mov Sci 2015; 40: 154-62.

39. Plante JE, Wikstrom EA. Differences in clinician-oriented outcomes among controls, copers, and chronic ankle instability groups. Phys Ther Sport 2013; 14: 221-6.

40. Garn SN, Newton RA. Kinesthetic awareness in subjects with multiple ankle sprains. Phys Ther 1988; 68: 1667-71.

41. Guskiewicz KM, Perrin DH. Research and clinical applications of assessing balance. J Sport Rehabil 1996; 5: 45-63.

42. Hall EA, Chomistek AK, Kingma JJ, Docherty CL. Balanceand strength-training protocols to improve chronic ankle instability deficits, part I: assessing clinical outcome measures. J Athl Train 2018; 53: 568-77.

43. Yamchi FR, Letafatkar A, Esmaeilpour S, Balance D. The effect of 8 weeks virtual reality training on static and dynamic balance and performance in male athletes 
with functional ankle instability. Phys Treat 2021; 11: 45-54.

44. Anguish B, Sandrey MA. Two 4-week balance-training programs for chronic ankle instability. J Athl Train 2018; 53: 662-71.

45. Spencer Cain M, Ban RJ, Chen YP, Geil MD, Goerger BM, Linens SW. Four-week ankle-rehabilitation programs in adolescent athletes with chronic ankle instability. J Athl Train 2020; 55: 801-10.

46. Cruz-Díaz D, Hita-Contreras F, Lomas-Vega R, OsunaPérez MC, Martínez-Amat A. Cross-cultural adaptation and validation of the Spanish version of the Cumberland Ankle Instability Tool (CAIT): an instrument to assess unilateral chronic ankle instability. Clin Rheumatol 2013; 32: 91-8.

47. O'Driscoll J, Kerin F, Delahunt E. Effect of a 6-week dynamic neuromuscular training programme on ankle joint function: a case report. Sport Med Arthrosc Rehabil Ther Technol 2011; 3: 13.

48. Schiftan GS, Ross LA, Hahne AJ. The effectiveness of proprioceptive training in preventing ankle sprains in sporting populations: a systematic review and meta-analysis. J Sci Med Sport 2015; 18: 238-44.

49. Gunel MK, Kara OK, Ozal C, Turker D. Virtual reality in rehabilitation of children with cerebral palsy. Cereb Palsy-Challenges Futur 2014; 273-301.

50. Khalil AA, Mohamed GA, Abd El Rahman SM, Elhafez SM, Nassif NS. Effect of Wiihabilitation on strength ratio of ankle muscles in adults. J Phys Ther Sci 2016; 28: 2862-6.

51. Manzoor S, Afzal F, Gulraiz Q, Khalid M. Effects of joints weight bearing exercises combine with range of motion exercises on affected limbs in patents with stroke. Int J Phys Med Rehabil 2017; 5: 410.

52. Punt IM, Armand S, Ziltener JL, Allet L. Effect of Wii Fit ${ }^{\top M}$ exercise therapy on gait parameters in ankle sprain patients: a randomized controlled trial. Gait Posture 2017; 58: 52-8.

53. Vernadakis N, Gioftsidou A, Antoniou P, Ioannidis D, Giannousi $M$. The impact of Nintendo Wii to physical education students' balance compared to the traditional approaches. Comput Educ 2012; 59: 196-205. 